\title{
The Effect of Sexism on the Acceptance of Homosexuality in Teenagers in China
}

\author{
Xinyue Miu ${ }^{1, a, ~}{ }^{*}, \dagger$, Diana Tsang ${ }^{2, *}{ }^{*}$, Zhuoxuan Wang ${ }^{3, *}{ }^{*}$, Xiyuan Zhang ${ }^{4, *, \dagger}$ \\ ${ }^{1}$ Changzhou university \\ ${ }^{2}$ Dulwich College Beijing \\ ${ }^{3}$ Hun School of Princeton \\ ${ }^{4}$ Guangdong experimental high school \\ *Corresponding author Email: ${ }^{a}$ guanghua.ren@gecacademy.cn \\ ${ }^{\dagger}$ These authors contributed equally.
}

\begin{abstract}
This research focuses on the sexual stereotype of teenagers in China. To find out the relation between sexism and homosexuality and the influence of traditional Chinese values, stereotypical gender norms, and biased social media on the acceptance of homosexuality of teenagers in modern Chinese society. Both qualitative and quantitative methods will be employed in this research. A survey has been designed and conducted in this research to better known the real situation and acceptance of homosexuality and stereotype from Chinese and the dynamic change of opinion under the influence of social media.
\end{abstract}

Keywords: Homosexuality, Stereotype, Teenagers, Survey, Culture.

\section{INTRODUCTION}

The Universal Declaration of Human Rights states, "all human beings are born free and equal in dignity and rights [1]." Yet, in various parts of the world, gay people are denied many rights. As of the moment, homosexual activity is illegal in 71 countries in the world, and 151 countries do not offer full protection for discrimination against the Lesbian, Gay, Bisexual, Transgender (LGBT) community, including China [2].

In China, the word homosexuality usually has a negative connotation. The deeply rooted societal values often cause this during the communist period after 1949 that everyone has filial duties and is responsible for continuing the bloodline of their families, which is unattainable for same-sex couples [3].

Teenagers are not an exception to such discriminations. From their peer pressure, such as having to act according to their sex stereotypically, those who do not often face backlashes. This phenomenon created a detrimental environment for the acceptance of homosexuality in students. Furthermore, traditional family values affect teenagers as well, where their family members, especially parents, often tell them to act like a girl or a boy by their parents. Other examples include the implications of filial pieties. The rejection of people acting out of their gender norms, in turn, affects the acceptance of homosexuality in society.

Thus, through our research, we would like to find out the relations between sexism and homosexuality, particularly the effect of traditional Chinese values, stereotypical gender norms, and biased social media on the acceptance of homosexuality of teenagers in modern Chinese society, to know how the relation affect homophobia and contribute to the liberation of human rights. Based on the literature review and further research in China, we hope to find out through our research that there is a strong correlation between stereotypical gender norms instituted by the different agents of socialization and homophobia.

Additionally, we have only included genders such as "boys" and "girls" in our study due to time limitations, and we do not wish to cause any offense to the other genders.

\section{LITERATURE REVIEW}

\subsection{Sexism's Negative Influence}

Gina Msaequemay points out the definition of sexism: one sex is superior to or more valuable than another sex [4]. It imposes limits on what men and boys 
can and should do and what women and girls should do, although the concept of sexism was initially formulated to raise consciousness about the oppression of girls and women [4]. The concept of sexism explains that prejudice and discrimination are based on sex or gender, not biological inferiority. The men's movement reasoned that it was time to document men's oppression [4]. According to Brandt, sexist ideologies for females are associated with a lower likelihood of voting for female political candidates, less support and occupational domains, and opposition to public policies designed to attenuate male dominance [5]. Leaper and Brown also pointed out that gender-based discrimination and sexism during adolescence can include bothering sexual harassment and gender bias in academic and athletic contexts [6]. Girls are often treated unfairly in nontraditional achievement contexts [6]. Many parents tend to have high expectations of sons over daughters in math, science, computers, and sports [6]. According to Leaper and Brown's research, Girls from 12-year-old to 18-year-old of varied socioeconomic and ethnic backgrounds completed surveys of personal experiences with sexual harassment, academic sexism (regarding science, math, and computer technology), and athletics [6]. Most girls reported sexual harassment (90\%), academic sexism (52\%), and athletic sexism (76\%) at least once, with the likelihood increasing with age [6]. Gordon suggests that in their writing "What Do We Say When We Hear 'Faggot'?" the stereotypical gender roles for males are much more rigid than those for females in the American society nowadays, which in turn causes homophobic remarks [7]. For instance, Gordon noted that a boy who was performing a stereotypical female task, including sewing or cooking, risks being called a "faggot" or "sissy" [7]. The finding suggests that while it is generally more acceptable for girls to perform tasks out of their "gender bubble," stigma still surrounds boys trying to do the same and possibly causes homophobia [7]. This phenomenon is also problematic since sexism toward boys causes gay students to become less accepted by their peers due to not conforming to the gender norms [7]. T. Digby, who has similar ideas with Gorden, even points out that while men are suffering from sexual inequality and second sexism. He believes that women have already acquired equality [8]. While men are victims of second sexism, they stand against "conservatives who endorse traditional gender roles" [8]. It is overwhelmingly men who discriminate against other men who are gay and intimidate and assault gays. The overwhelming majority of anti-male sexual harassment cases are male and nominally heterosexual [8]. When men get some benefits from the stereotype of males' role in society, men do not believe that it is discrimination because the advantages of sexism are not unfair and even beneficial for men. Only when men are suffering from the disadvantages of discrimination will men realize that what they have experienced is a form of discrimination, like sexism for men [8].

\subsection{Basic Reasons for Chinese's Exclusion toward Homosexuality}

First, the traditional cultural ideas toward homosexuality strongly affected the Chinese and made Chinese people comparatively more conservative and unacceptable to homosexuality. John Choe emphasizes Confucianism's impacts on Asian values and Asian attitudes toward homosexuality, resulting in the perpetuation of homophobic laws [9]. Confucianism in Asia was essentially invoked as a set of stripped-down ethical values that had a specific role in the state's service [9]. As a generalized moral philosophy, Confucianism entailed respect for social authority [9]. In this climate, homosexuality was marginalized and rendered invisible [9]. Only rarely did homosexuality make it into the interstices of public discourse, and even then, homosexuality was portrayed negatively as a "mental illness" or a "relatively rare occurrence" in Chinese societies [9]. Second, the lack of laws from the government makes homosexuals' situation more difficult and makes it Chinese people feel hard to accept the LGBTQ community. Zhang, Li, Wang, Wang, and Liu agree with Choe's opinions about the influence of traditional cultures toward Chinese's thoughts about homosexuality: due to the traditional culture, old rules, and Chinese morals, it is hard for Chinese people and government to accept homosexuality and publish laws to protect them immediately [10]. Although the Chinese government cannot publish some laws directly to protect LGBTQ, they can use some other rules to replenish today's laws and gradually make people accept homosexuality [10]. For instance, the Chinese government can add a new rule that homosexuality is given the right to marry heterosexuals after proving that they live together [10]. The condition of homosexuality in China is well-described that most Chinese cannot accept homosexuality. Still, eventually, this situation can be changed with the time passed and the promulgation of new laws [10]. Third, the lack of education about sex and LGBTQ leads people to not understand LGBTQ but even result in people's misunderstanding of homosexuality. Ruili Huang talks about how some Chinese textbooks describe homosexuality as a mental disorder or a disease [11]. Although homosexuality is not considered a mental disorder or disease in Chinese government documents, the general lack of knowledge still hugely misleads people that homosexuality is an illness and should not be accepted [11]. These three factors actually are mutually reinforcing each other to accumulate the ingrained Chinese' exclusion toward homosexuality.

\section{RESEARCH METHODOLOGY}

We will mainly collect information by conducting combinations of quantitative and qualitative surveys supplemented by consulting various documents. For this social topic, more first-hand materials are needed. 
Qualitative surveys can give more true feedback on the current situation of homosexuality and sexism in Chinese society.

The first part is the survey. To ensure that our survey is quantitative, the most crucial thing in the survey is selecting samples and setting the survey content. The selection of quantitative survey samples should consider the geographic region, age level, economic development level, education level of the people. One is the selection of regions. The choice of regions should be reasonable. The second is selecting age groups to make the research results more authentic and credible. Teenagers of every age must be chosen. For example, young people may be more accepting of homosexuality, and fewer people have sexist ideas. Older people are very different. The third is to consider the degree of economic development and education. There are often more people with higher education and more advanced thinking in economically developed places. The fourth is to consider the issue of religious beliefs. Different religions have different views on homosexuality and gender. Most people who believe in religions are more religious. Their religion largely directly determines their views on homosexuality and gender.

The biggest problem is that it is difficult for us to find diverse interviewees from different areas and generations. Survey responses are mostly from young people in big cities, which means that they have many more opportunities to get in touch with the Internet and know and meet gay people. As our research target is teenagers, we limit our participants to teenagers living in cities. Although we can interview some older generations around us to know about their ideas toward homosexuality, this online survey also restricts our target volunteers familiar with the Internet. This may strongly affect our results because our friends are more open to homosexuality and do not know traditional sexism.

\subsection{Survey Design}

Our survey has asked respondents multiple choice questions, scale questions, and open-ended questions on their stance toward homosexuality and their experiences with gender stereotypes. In the four sections, questions include their demographic, whether they identify as part of the LGBT community, to what extent do they accept homosexuality, whether they are personally acquainted with someone that is homosexual, and scenarios such as what they would do if your favorite celebrity or family members comes out as homosexual. Lastly, the survey also includes photos for the respondents to rate how gay they think the person in the image is.

\subsubsection{Demographic}

This section includes questions regarding the respondents' age, sex, gender, ethnicity, education, and religion. This allows us to better understand the respondents as individual people and how their different demographical backgrounds may show trends and explain the differences in responses.

\subsubsection{Homosexuality}

In this section, the questions mostly include their acceptance of homosexuality, the reasons behind it, and scenarios to gauge how they react to someone coming out as gay. It helps us know the many different factors that have affected their level of acceptance. It allows us to look deeper into each factor, thus taking our research to a more specific area and corroborating our conclusions made from the literature review.

\subsubsection{Genders}

This section touches on the issues of gender and how they are presented to the respondents themselves. It allows us to know how sexism appears in everyday life and helps us find the correlation between gender stereotypes and homosexuality.

\subsubsection{Photo Questions}

In Figure 1, a stereotypical gay person is shown. The person is wearing makeup and touching his lips with his hands. Figure 2 contains a photo of a stereotypical legitimate "heterosexual" Chinese man. He is wearing a pressed shirt. In Figure 3, how people perceive homosexuality in females is investigated. This is an image of a very stereotypical sweet and girly Chinese female. In figure 4, a photo of a "tomboy" girl was shown.

This section allows us to see how the respondents indirectly react toward different gender stereotypes through a more passive method of choosing how "gay" the person presented in the photo is rather than asking them directly. This could tell us how people genuinely react to gender stereotypes and how that can be linked to the perception of homosexuality. 


\section{PRELIMINARY RESULTS}

\subsection{Key Findings and Analysis}

Out of the 52 respondents who responded to our survey, 3.8\% identifies as part of the LGBT community, along with 9.6\% "maybe." Overall, the acceptance of homosexuality is 5.69 out of 10 . Certain factors that affected people's acceptance of homosexuality include watching pride parades, celebrities being a part of or supporting the LGBT community, and increasing community awareness through the news, social media, and films. Most suggested that their acceptance of homosexuality has not changed over time. Furthermore, $34.6 \%$ of the respondents claimed to be personally acquainted with someone who is gay. However, no one has a family member that is gay, and $44.2 \%$ are not acquainted with any, either. In addition, $69.6 \%$ do not

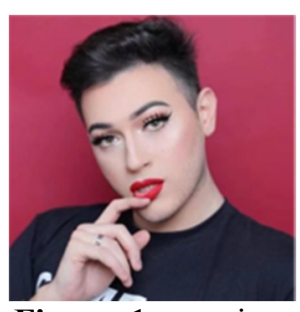

Figure. 1. a. a sissy man

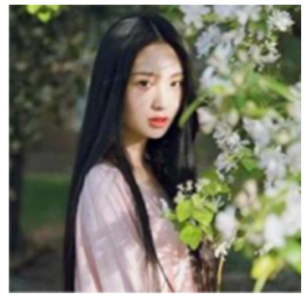

Figure. 1. c. a sissy woman

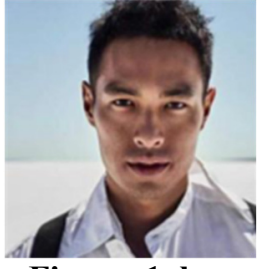

Figure. 1. b. a manly man

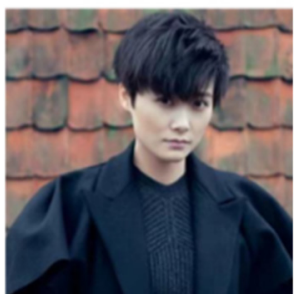

Figure. 1. d. a manly woman want to become acquainted with them at all. When asked about what they would do if their favorite celebrity comes out as gay, a similar amount of people said they would congratulate them, do nothing, or stop following them. When asked about what they would do if their (hypothetical) child comes out as gay, most responses were positive. However, a few suggested that they would beat their child and deny their homosexuality.

\subsection{Photo Questions}

From the results for Figure 1, there is a trend that most people think that he is extremely gay. Only eight people chose to tell whether he is gay or not, showing that they are not judging his homosexuality by his looks. For Figure 2, there is a positive correlation that most people think that he is not very gay, and many people have chosen that they cannot tell whether he is gay or not. The contrast between the reactions toward the two males certainly shows that some people are whether others are gay based on how much they fit with their gender norms.

In Figure 3, once again, most people choose that they cannot tell whether she is gay or not. However, in Figure 4, many people have chosen that they cannot tell once again. By itself, this should be a positive reaction to a person who looks, stereotypical lesbian. However, as we have seen from the first image, people who said the person looked extremely gay just by the way he looks. This raises the question as to why females are not considered to be able to be gay.

\section{ANALYSIS}

As mentioned in other parts, homophobia in China has been constant for many years. Many homosexual people are suffering from social discrimination, lack of laws protection, and lack of approval. Without related laws and regulations, the group of homosexuality cannot protect themselves from discrimination; or even worse, they will be regarded as people who suffer from mental disease. Thus, through our research, we would like to find out the relations between sexism and homosexuality, particularly traditional Chinese values, stereotypical gender norms, and biased social media. It affects teenagers on the acceptance of homosexuality in modern Chinese society, to know how the relation affect homophobia and contribute to the liberation of human rights. Furthermore, we attempted to show that homophobia can be affected by the sexism to the homosexuality teams, due to the deeply rooted societal values, in our study. Surprisingly, Digby [8] points out that men suffer more from sexual inequality and second sexism than women. What is more, Chinese laws affect the Chinese as well. There are no related laws that protect homosexuality's rights before the Chinese government considers the citizens have enough acceptance of homosexuality in Zhang's article[10]. The study from Miller [12] shows how social media impact gender and considers the social media bring people more freedom and a tolerant environment for gay people. Interestingly, our current survey shows that the 52 interviewees' acceptance of homosexuality is about 5.69 when the most acceptance level is at 10 . Overall, certain factors that affected people's acceptance of homosexuality include watching pride parades, celebrities being a part of or supporting the LGBT community, and increasing awareness of this community through the news, social media, and films. Most suggested that their acceptance of homosexuality has changed over time. Zhang's [10] study also said that there are some laws to prove the relationship identity of homosexuality. However, many target volunteers still consider homosexuality as not a natural sexual orientation in our survey data. According to our survey, about 69.9 percent of people even do not want to meet homosexuality, and most of their answers are in disagreement when they are asked their reaction 
after they know their relatives are homosexual. One of the facts of homosexuality is that they cannot have their biological children, which means they cannot carry on their family names (family lines). This contradicts the Chinese traditional rituals and cannot be accepted by the older generations. Hence, our research would serve as proof of the concept that homophobia in China is affected by traditional Chinese values, stereotypical gender norms, and social media. It still needs more time to complement the liberation of homosexuality in China and for all Chinese people to have homosexuality acceptance and a deep understanding of it. Although a qualitative survey gives us more opportunities to find out volunteers' real thoughts about this topic, there are also some limitations of our study that we cannot ignore. Because of limited time and words, and without going through the official papers and the ambiguous attitudes from the government, our research can only base on the public thoughts and attitudes, which may be incomplete (not enough) for this topic. In addition, the number of people who have done our survey is not enough because China has 1400 million people.

\section{CONCLUSION}

In conclusion, although China's society is continually improving, not only at the acceptance but also at the tolerance of new things, there are still many counter views with the homosexuality that against traditional cultural and public recognition. In this study, we have developed a survey where people from different age groups wrote their answers about the questions related to homosexuality. By analyzing the survey results and finding other studies, the evidence suggests a strong correlation between stereotypical gender norms agents of socialization. This study provides additional evidence with respect to most Chinese have more acceptance of homosexuality, when the stereotype is decreasing in Chinese daily languages, social media, laws, etc., which has been mentioned both in our studies and references. Our group aims to help today's society accept varied kinds of sexual orientations through doing our studies. People should have the rights they deserved, like the legalization of gay marriage and the freedom of choosing sexual orientation, no matter their sexual preference. Further work is required to establish a clearer survey, search for more Chinese government information, and use varied ways to obtain data.

\section{REFERENCES}

[1] United Nations. (1948, December 10). Universal Declaration of Human Rights. United Nations. https:/www.un.org/en/universal-declarationhuman-rights/index.html.

[2] Equaldex. (2021, February 17). LGBT Rights in China. https://www.equaldex.com/region/china.
[3] Steward, W. T., Miège, P., \& Choi, K.-H. (2013). Charting a Moral Life: The Influence of Stigma and Filial Duties on Marital Decisions among Chinese Men who Have Sex with Men. PLOS ONE, 8(8). https://doi.org/10.1371/journal.pone.0071778

[4] Thompson, N. (1995). Men and Anti-Sexism. The British Journal of Social Work, 25 (4), 459-475, Retrieved June 3, 2021, from http://www.jstor.org/stable/23709801

[5] Brandt, M. (2011). Sexism and Gender Inequality Across 57 Societies. Psychological Science, 22(11), 1413-1418. Retrieved March 10, 2021, from http://www.jstor.org/stable/41320046

[6] Leaper, C., \& Brown, C. (2008). Perceived Experiences with Sexism among Adolescent

[7] Gordon, L. (2016). Rethinking our classrooms, Volume 1: Teaching for equity and justice. In W. Au, B. Bigelow, \& S. Karp (Eds.), Rethinking our classrooms, volume 1: teaching for equity and justice (pp. 86-87). essay, Zenescope Entertainment.

[8] Digby, T. (2003). Male Trouble: Are Men Victims of Sexism? Social Theory and Practice, 29(2), 247-273. Retrieved February 14, 2021, from http://www.jstor.org/stable/23559076

[9] Choe, J. (2014). Gay and Lesbian Rights in Confucian Asia: The Cases of Hong Kong, Singapore, and Taiwan. Gay and Lesbian Rights in Confucian Asia: The Cases of Hong Kong, Singapore, and Taiwan. Retrieved January 01, 2014, from http://repository.upenn.edu/curej/172

[10] Zhaofeng Zhang, Junfeng Li, Mingying Wang, Zhina Wang and Haoran Liu. (2017) The Exploration of the Living Conditions of Gays in Today's China. From https://kns.cnki.net/kcms/detail/detail.aspxdbcode= CJFD\&dbname $=$ CJFDLAST2018 \& finame $=$ JMSA $201723275 \& \mathrm{v}=0 \mathrm{jP} 3 \mathrm{kOSPT} c \mathrm{mnqkkC8ZI3jm4x886}$ qCcj5rMUWfC7xAw16yW2QqFPrK5Fr8IjILL2.

[11] Ruili Huang. (Oct, 28, 2020) China's stance on homosexuality has changed. Its textbooks haven't, from

https://www.nytimes.com/2020/10/28/business/int ernational/china-gay-homoexuality-textbookslawsuit.html

[12] Miller, D., Costa, E., Haynes, N., McDonald, T., Nicolescu, R., Sinanan, J.Wang, X. (2016). Gender. In How the World Changed Social Media (pp. 114-127). London: UCL Press. Retrieved February 14, 2021, from http://www.jstor.org/stable/j.cttlg69z35.15 